\title{
Statistical correlation for a three-terminal normal-metal-superconductor- superconductor hybrid system
}

\author{
Baigeng Wang and Jian Wang* \\ Department of Physics, The University of Hong Kong, Pokfulam Road, Hong Kong, China \\ (Received 16 May 2002; revised manuscript received 13 September 2002; published 14 January 2003)
}

\begin{abstract}
It is well known that the particles in a beam of bosons obeying Bose-Einstein statistics tend to cluster (bunching effect), while the particles in a degenerate beam of fermions obeying Fermi-Dirac statistics expel each other (antibunching effect). Here we investigate the statistical correlation effect for a three-terminal normal-metal-superconductor-superconductor hybrid mesoscopic system. By using a nonequilibrium Green'sfunction technique, we obtain a positive cross correlation when the external voltage is smaller than the gap energy, which demonstrates bosonic behavior. In the larger voltage limit, the cross correlation becomes negative due to the contribution of the quasiparticles. At large voltages, the oscillation between fermionic and bosonic behavior of cross correlation is also observed in the strong-coupling regime as one changes the position of the resonant levels.
\end{abstract}

DOI: 10.1103/PhysRevB.67.014509

PACS number(s): 74.50. $+\mathrm{r}, 72.70 .+\mathrm{m}, 74.40 .+\mathrm{k}, 73.23 .-\mathrm{b}$

\section{INTRODUCTION}

There are two kinds of quantum statistics in nature. All particles have either half-integral or integral spin (in units of the Planck constant $\hbar$ ) and they obey Fermi-Dirac or BoseEinstein statistics, respectively. It is also noted ${ }^{1}$ that there is an effective attraction between the bosons and an effective repulsion between the fermions. These are the well-known statistical correlation effects, ${ }^{2}$ which are purely quantum effects. The experiments examining the quantum statistical properties date back to the pioneering work by Hanbury Brown and Twiss (HBT). ${ }^{3}$ They used photon intensity interferometry to probe the intensity correlation information between two partial beams, which was generated by a beam splitter. Due to the bosonic property of photons, the positive intensity correlation was observed, indicating an enhanced probability for the simultaneous detection of two photons, one in each partial beam. This means that photons tend to bunch in clusters. Several theoretical works have suggested the different analogies of this experiment with electrons in mesoscopic systems. The fermionic analog of HBT experiments, one by Henny et al. ${ }^{4}$ and the other by Oliver et al., ${ }^{5}$ showed the expected negative intensity correlation and observed the antibunching effect. On the theoretical side, when Torriès and Martin ${ }^{6}$ investigated a three-terminal normalmetal-superconductor-superconductor (N-N-S) mesoscopic system, both positive and negative correlations were found in the Andreev regime. Very recently, Samuelsson and Büttiker ${ }^{7}$ studied the same structure and found the positive correlation for a wide range of junction parameters which survives even in the absence of the proximity effect. The statistics of charge transport of a three-terminal N-N-S beam splitter has also been investigated ${ }^{8}$ and positive cross correlation is found between the currents in two normal leads for a wide parameter range. Instead of the structures of Refs. 6-8, here we consider a three-terminal mesoscopic N-S-S hybrid system. This structure is a direct photon analogy of the HBT interferometer which has a normal lead and two supercon- ducting leads. A quantum dot, connected by these three terminals, acts as a splitter. Suppose that the chemical potentials $\mu_{s}$ for both superconducting reservoirs are set to zero, and the chemical potential for the normal is above zero, i.e., $e V$ $>0$, which guarantees the electron current passing from the normal lead to both superconducting leads. We further assume the temperature is very low. If the external voltage $e V$ is smaller than the gap energy $\Delta$ of the superconducting leads, the single-quasiparticle current is forbidden. In this case, we only have a two-electron current due to the presence of the Andreev reflection process, i.e., incoming electrons being Andreev reflected into outgoing holes with the transfer of a Cooper pair into the superconductor. This means that an electron (with energy $\epsilon$ above the Fermi level and spin $\sigma$ ) in the normal lead has to combine with another electron (with energy $-\epsilon$, below the Fermi level and spin $-\sigma$ ) to pass through the normal-metal-superconductor interface. Does this electron pair look like a boson? Or rather, can we obtain a positive cross correlation function $\left\langle\Delta I_{\alpha} \Delta I_{\beta}\right\rangle$ with $\alpha \neq \beta$ ) between two superconducting leads? The purpose of this paper is to answer this question. We note that due to the current conservation the cross correlation function of a two-lead system must be negative regardless of normal or superconducting leads. Instead of considering the fluctuation in a single electron beam through the two-lead system, the HBT experiment considered here focuses on the cross correlation of two beams from the beam splitter. Hence we expect positive cross correlation at small voltages which is indeed what we found in this work. When $e V>\Delta$, the quasiparticles will also participate in the transport. Due to the fermionic nature of quasiparticles, it will partially cancel the positive contribution of the electron pair to the cross correlation. The competition of these two contributions from the electron pair and quasiparticles can lead to either positive or negative cross correlation, depending on which contribution dominates.

\section{THEORETICAL FORMULATION}

We begin with the following model Hamiltonian: 


$$
\begin{aligned}
H= & \sum_{p} \epsilon_{p} C_{1, p \sigma}^{+} C_{1, p \sigma}+\sum_{k n}\left[\sum_{\sigma} \epsilon_{k} C_{n, k \sigma}^{+} C_{n, k \sigma}\right. \\
& \left.+\Delta C_{n, k \uparrow}^{+} C_{n,-k \downarrow}^{+}+\Delta C_{n,-k \downarrow} C_{n, k \uparrow}\right]+\sum_{\sigma} \epsilon_{0} d_{\sigma}^{+} d_{\sigma} \\
& +\sum_{p \sigma}\left[T_{1, p} C_{1, p \sigma}^{+} d_{\sigma}+\text { c.c. }\right]+\sum_{k n \sigma}\left[T_{n, k} C_{n, k \sigma}^{+} d_{\sigma}+\text { c.c. }\right],
\end{aligned}
$$

where the first term denotes the Hamiltonian of the normal lead. The second term $(n=2,3)$ describes the Hamiltonian of two BCS superconducting leads. Here $C_{1, k \sigma}^{\dagger}$ is the creation operator of electrons in the normal lead and $C_{n, k \sigma}^{\dagger}$ is the corresponding creation operator in the superconducting lead. The third term is the Hamiltonian for a quantum dot, which is used to mimic a tunable beam splitter. Here we have applied a gate voltage which can control the level of the dot so that $\epsilon_{0}=\epsilon_{0}^{(0)}+e v_{g}$. Without loss of generality, we set $\epsilon_{0}^{(0)}$ $=0$. The other terms in Eq. (1) are Hamiltonians describing the couplings between the quantum dot and leads. To simplify the discussion, we have assumed that two superconducting leads have the same gap energy $\Delta$. We have also neglected the supercurrent between two superconducting leads ${ }^{9}$ and assumed that the hopping matrix elements are independent of the spin index.

In the following, we will calculate the cross correlation between two partial beams through two superconducting leads. The current operator for the superconducting lead 2 or 3 is

$$
\hat{I}_{\alpha}=\hat{I}_{\alpha \uparrow}(t)+\hat{I}_{\alpha \downarrow}(t)
$$

with

$\hat{I}_{\alpha \sigma}(t)=i e\left[\sum_{k} C_{\alpha, k \sigma}^{\dagger} C_{\alpha, k \sigma}, H\right]=i e \sum_{k}\left[T_{\alpha k} C_{\alpha, k \sigma}^{+} d_{\sigma}-\right.$ c.c. $]$

where $\alpha=2,3$. Due to the electron-hole symmetry of the system, we have $\hat{I}_{\alpha \uparrow}(t)=\hat{I}_{\alpha \downarrow}(t)$. Hence the current operator can be rewritten as

$$
\hat{I}_{\alpha}(t)=2 i e \sum_{k}\left[T_{\alpha k} C_{\alpha, k \uparrow}^{+} d_{\uparrow}-\text { c.c. }\right] \text {. }
$$

The cross correlation between two superconducting leads is defined as

$$
P_{23} \equiv\left\langle\Delta I_{2}\left(t_{1}\right) \Delta I_{3}\left(t_{2}\right)\right\rangle \equiv\left\langle\left[\hat{I}_{2}\left(t_{1}\right)-\bar{I}_{2}\right]\left[\hat{I}_{3}\left(t_{2}\right)-\bar{I}_{3}\right]\right\rangle
$$

with $\bar{I}_{\alpha} \equiv\left\langle\hat{I}_{\alpha}\right\rangle$. Here $\langle\ldots\rangle$ denotes both the statistical average and quantum average on the nonequilibrium state. Using the expression of the current operator, the cross correlation between two superconducting leads is

$$
\begin{aligned}
P_{23}\left(t_{1}, t_{2}\right)= & -4 e^{2} \sum_{k, k^{\prime}}\left[T_{2, k} T_{3, k^{\prime}} G_{d \uparrow k \uparrow}^{<}(2,1) G_{d \uparrow k^{\prime} \uparrow}^{>}(1)\right. \\
& +T_{2, k}^{*} T_{3, k^{\prime}}^{*} G_{k^{\prime} \uparrow d \uparrow}^{<}(2,1) G_{k \uparrow d \uparrow}^{<}(1,2) \\
& -T_{2, k} T_{3, k^{\prime}}^{*} G_{k^{\prime} \uparrow k \uparrow}^{<}(2,1) G_{d \uparrow d \uparrow}^{>}(1,2) \\
& \left.-T_{2, k}^{*} T_{3, k^{\prime}} G_{d \uparrow d \uparrow}^{<}(2,1) G_{k \uparrow k^{\prime} \uparrow}^{>}(1,2)\right],
\end{aligned}
$$

where we have used the abbreviation $G\left(t_{1}, t_{2}\right)=G(1,2)$ and we have used $k$ and $k^{\prime}$ to label, respectively, the second and third superconducting leads. The Green's functions $\mathbf{G}^{r, a,<,>}$ in a $2 \times 2$ Nambu representation take the following forms: ${ }^{10-12}$

$$
\begin{aligned}
& G_{\alpha \beta}^{r, a}\left(t_{1}, t_{2}\right) \\
&= \mp i \theta\left( \pm t_{1} \mp t_{2}\right) \\
& \times\left(\begin{array}{cc}
\left\langle\left\{X_{\alpha \uparrow}\left(t_{1}\right), Y_{\beta \uparrow}^{+}\left(t_{2}\right)\right\}\right\rangle & \left\langle\left\{X_{\alpha \uparrow}\left(t_{1}\right), Y_{\beta \downarrow}\left(t_{2}\right)\right\}\right\rangle \\
\left\langle\left\{X_{\alpha \downarrow}^{+}\left(t_{1}\right), Y_{\beta \uparrow}^{+}\left(t_{2}\right)\right\}\right\rangle & \left\langle\left\{X_{\alpha \downarrow}^{+}\left(t_{1}\right), Y_{\beta \downarrow}\left(t_{2}\right)\right\}\right\rangle
\end{array}\right), \\
& G_{\alpha \beta}^{<}\left(t_{1}, t_{2}\right)=i\left(\begin{array}{cc}
\left\langle Y_{\beta \uparrow}^{+}\left(t_{2}\right) X_{\alpha \uparrow}\left(t_{1}\right)\right\rangle & \left\langle Y_{\beta \downarrow}\left(t_{2}\right) X_{\alpha \uparrow}\left(t_{1}\right)\right\rangle \\
\left\langle Y_{\beta \uparrow}^{+}\left(t_{2}\right) X_{\alpha \downarrow}^{+}\left(t_{1}\right)\right\rangle & \left\langle Y_{\beta \downarrow}\left(t_{2}\right) X_{\alpha \downarrow}^{+}\left(t_{1}\right)\right\rangle
\end{array}\right), \\
& G_{\alpha \beta}^{>}\left(t_{1}, t_{2}\right)=-i\left(\begin{array}{cc}
\left\langle X_{\alpha \uparrow}\left(t_{1}\right) Y_{\beta \uparrow}^{+}\left(t_{2}\right)\right\rangle & \left\langle X_{\alpha \uparrow}\left(t_{1}\right) Y_{\beta \downarrow}\left(t_{2}\right)\right\rangle \\
\left\langle X_{\alpha \downarrow}^{+}\left(t_{1}\right) Y_{\beta \uparrow}^{+}\left(t_{2}\right)\right\rangle & \left\langle X_{\alpha \downarrow}^{+}\left(t_{1}\right) Y_{\beta \downarrow}\left(t_{2}\right)\right\rangle
\end{array}\right),
\end{aligned}
$$

where $X$ and $Y$ stand for the annihilation operators, such as $C_{1, p}, C_{n, k}$, and $d$. These Green's functions satisfy the general relation $\mathbf{G}^{>}=\mathbf{G}^{<}+\mathbf{G}^{r}-\mathbf{G}^{a}$. Using the Keldysh equation $^{13}$

$$
\mathbf{G}^{<,>}=\left(1+\mathbf{G}^{r} \boldsymbol{\Sigma}^{r}\right) \mathbf{G}_{0}^{<,>}\left(1+\boldsymbol{\Sigma}^{a} \mathbf{G}^{a}\right)+\mathbf{G}^{r} \boldsymbol{\Sigma}^{<} \mathbf{G}^{a}
$$

we have the following relations:

$$
\begin{aligned}
& G_{d \uparrow k \uparrow}^{<,>}\left(t_{1}, t_{2}\right)=T_{2, k}^{*} \int d t\left[G_{d \uparrow d \uparrow}^{r}\left(t_{1}, t\right) g_{k \uparrow k \uparrow}^{<,>}\left(t, t_{2}\right)\right. \\
& +G_{d \uparrow d \uparrow}^{<,>}\left(t_{1}, t\right) g_{k \uparrow k \uparrow}^{a}\left(t, t_{2}\right) \\
& +G_{d \uparrow d \downarrow}^{r}\left(t_{1}, t\right) g_{k \downarrow k \uparrow}^{<,>}\left(t, t_{2}\right) \\
& \left.+G_{d \uparrow d \downarrow}^{<,>}\left(t_{1}, t\right) g_{k \downarrow k \uparrow}^{a}\left(t, t_{2}\right)\right], \\
& G_{k \uparrow d \uparrow}^{<,>>}\left(t_{1}, t_{2}\right)=T_{2, k} \int d t\left[g_{k \uparrow k \uparrow}^{<,>>}\left(t_{1}, t\right) G_{d \uparrow d \uparrow}^{a}\left(t, t_{2}\right)\right. \\
& +g_{k \uparrow k \uparrow}^{r}\left(t_{1}, t\right) G_{d \uparrow d \uparrow}^{<,>>}\left(t, t_{2}\right) \\
& +g_{k \uparrow k \downarrow}^{<,>}\left(t_{1}, t\right) G_{d \downarrow d \uparrow}^{a}\left(t, t_{2}\right) \\
& \left.+g_{k \uparrow k \downarrow}^{r}\left(t_{1}, t\right) G_{d \downarrow d \uparrow}^{<,>}\left(t, t_{2}\right)\right], \\
& G_{k \uparrow k^{\prime} \uparrow}^{<,>}\left(t_{1}, t_{2}\right)=T_{3, k^{\prime}}^{*} \int d t\left[G_{k \uparrow d \uparrow}^{r}\left(t_{1}, t\right) g_{k^{\prime} \uparrow k^{\prime} \uparrow}^{<,>}\left(t, t_{2}\right)\right. \\
& +G_{k \uparrow d \uparrow}^{<,>>}\left(t_{1}, t\right) g_{k^{\prime} \uparrow k^{\prime} \uparrow}^{a}\left(t, t_{2}\right) \\
& +G_{k \uparrow d \downarrow}^{r}\left(t_{1}, t\right) g_{k^{\prime} \downarrow k^{\prime} \uparrow}^{<,>}\left(t, t_{2}\right) \\
& \left.+G_{k \uparrow d \downarrow}^{<,>}\left(t_{1}, t\right) g_{k^{\prime} \downarrow k^{\prime} \uparrow}^{a}\left(t, t_{2}\right)\right],
\end{aligned}
$$


where $G_{k \uparrow, d \sigma}^{r}$ is given by

$$
\begin{aligned}
G_{k \uparrow d \sigma}^{r}\left(t_{1}, t_{2}\right)= & T_{2, k} \int d t\left[g_{k \uparrow k \uparrow}^{r}\left(t_{1}, t\right) G_{d \uparrow d \sigma}^{r}\left(t, t_{2}\right)\right. \\
& \left.+g_{k \uparrow k \downarrow}^{r}\left(t_{1}, t\right) G_{d \downarrow d \sigma}^{r}\left(t, t_{2}\right)\right] .
\end{aligned}
$$

Substituting the above relations into Eq. (1) and taking the Fourier transform $\left[P_{23}=\int d t_{1} d t_{2} P_{23}\left(t_{1}, t_{2}\right)\right]$, we obtain

$$
\begin{aligned}
P_{23}= & -4 e^{2} \Gamma_{2} \Gamma_{3} \int \frac{d E}{2 \pi}\left(\left(\mathbf{G}^{r} \mathbf{g}^{<}+\mathbf{G}^{<} \mathbf{g}^{a}\right)_{\uparrow \uparrow}\left(\mathbf{G}^{r} \mathbf{g}^{>}+\mathbf{G}^{>} \mathbf{g}^{a}\right)_{11}\right. \\
& +\left(\mathbf{g}^{<} \mathbf{G}^{a}+\mathbf{g}^{r} \mathbf{G}^{<}\right)_{11}\left(\mathbf{g}^{>} \mathbf{G}^{a}+\mathbf{g}^{r} \mathbf{G}^{>}\right)_{11} \\
& -G_{11}^{>}\left\{\left(\mathbf{g}^{r} \mathbf{G}^{r} \mathbf{g}^{<}\right)_{11}+\left[\left(\mathbf{g}^{<} \mathbf{G}^{a}+\mathbf{g}^{r} \mathbf{G}^{<}\right) \mathbf{g}^{a}\right]_{11}\right\} \\
& \left.\left.-G_{11}^{<}\left(\mathbf{g}^{r} \mathbf{G}^{r} \mathbf{g}^{>}\right)_{11}+\left[\left(\mathbf{g}^{>} \mathbf{G}^{a}+\mathbf{g}^{r} \mathbf{G}^{>}\right) \mathbf{g}^{a}\right]_{11}\right\}\right),
\end{aligned}
$$

where $\Gamma_{\alpha}=2 \pi \Sigma_{k} \rho_{N \alpha}\left|T_{\alpha k}\right|^{2}$ with $\alpha=2,3$ the linewidth functions. Here $\rho_{N 2,3}$ are the normal density of states of the superconducting leads 2 and 3 . We have used the wide-band limit ${ }^{14}$ and thus the linewidth function is independent of the energy. $\mathbf{G}^{r, a,<,>} \equiv \mathbf{G}_{d, d}^{r, a,<,>}$ are the full Green's functions for the quantum dot in the presence of the leads, while $\mathbf{g}^{r, a,<,>}$ are the exact Green's functions for the BCS superconductor in the absence of the coupling between the leads and quantum dot. Equation (7) is the central result of this paper. It describes the cross correlation for a three-terminal hybrid $\mathrm{N}-\mathrm{S}-\mathrm{S}$ system and is at any temperature and finite voltage, i.e., valid for both $e V \geqslant \Delta$ and $e V<\Delta$. In order to calculate this correlation, one must know all the Green's functions. The exact Green's functions $\mathbf{g}^{r, a,<}$ for the isolated superconducting leads are ${ }^{15,16}$

$$
\begin{gathered}
\mathbf{g}^{r}(E)=-\frac{i \zeta(E)}{2 \sqrt{E^{2}-\Delta^{2}}}\left(\begin{array}{ll}
E & \Delta \\
\Delta & E
\end{array}\right)=\left[\mathbf{g}^{a}(E)\right]^{+}, \\
\mathbf{g}^{<}(E)=i f(E) \theta(|E|-\Delta) \frac{\zeta(E)}{\sqrt{E^{2}-\Delta^{2}}}\left(\begin{array}{cc}
E & \Delta \\
\Delta & E
\end{array}\right),
\end{gathered}
$$

where $f(E)=1 /\left\{\exp \left[\beta\left(E-E_{F}\right)\right]+1\right\}$ is the well-known Fermi distribution function, $\theta(x)$ is the step function, and $\zeta(E)$ $=1$ when $E>-\Delta$, otherwise $\zeta(E)=-1$. We will choose the Fermi energy of the normal lead in line with the chemical potential $\mu_{s}$ of the superconducting condensate which is set to zero, i.e., $E_{F}=\mu_{s}=0$. The retarded Green's function for the quantum dot can be calculated using the Dyson equation

$$
\mathbf{G}^{r}(E)=\frac{1}{\left[\mathbf{G}_{0}^{r}(E)\right]^{-1}-\mathbf{\Sigma}^{r}(E)}
$$

with

$$
\mathbf{G}_{0}^{r}(E)=\frac{1}{\left(\begin{array}{cc}
E-\epsilon_{0} & 0 \\
0 & E+\epsilon_{0}
\end{array}\right)}
$$

and

$$
\boldsymbol{\Sigma}^{r}(E)=-\frac{i}{2} \Gamma_{1}\left(\begin{array}{ll}
1 & 0 \\
0 & 1
\end{array}\right)-\frac{i}{2}\left(\Gamma_{2}+\Gamma_{3}\right) \frac{\zeta(E)}{\sqrt{E^{2}-\Delta^{2}}}\left(\begin{array}{cc}
E & \Delta \\
\Delta & E
\end{array}\right) .
$$

The lesser Green's function can be obtained from the Keldysh equation $\mathbf{G}^{<}=\mathbf{G}^{r} \mathbf{\Sigma}^{<} \mathbf{G}^{a}$. Here the lesser self-energy is given by

$$
\begin{aligned}
\boldsymbol{\Sigma}^{<}(E)= & i \Gamma_{1}\left(\begin{array}{cc}
f(E+e V) & 0 \\
0 & f(E-e V)
\end{array}\right) \\
& +i f(E) \theta(|E|-\Delta) \frac{\Gamma_{2}+\Gamma_{3}}{\sqrt{E^{2}-\Delta^{2}}} \zeta(E)\left(\begin{array}{ll}
E & \Delta \\
\Delta & E
\end{array}\right) .
\end{aligned}
$$

Let us first consider the case in which external voltage is smaller than the gap energy and consider zero-temperature behavior so that there are no quasiparticles participating in the transport. In this case, only a two-electron current exists, i.e., the currents from incoming electrons and the Andreevreflected hole, and we have $\mathbf{g}^{r}=\mathbf{g}^{a}$ and $\mathbf{g}^{<,>}=0$, using the fact that

$$
\mathbf{G}^{<}=i \Gamma_{1} \mathbf{G}^{r}\left(\begin{array}{cc}
f_{+} & 0 \\
0 & f_{-}
\end{array}\right) \mathbf{G}^{a}
$$

and

$$
\mathbf{G}^{>}=i \Gamma_{1} \mathbf{G}^{r}\left(\begin{array}{cc}
f_{+}-1 & 0 \\
0 & f_{-}-1
\end{array}\right) \mathbf{G}^{a} .
$$

Equation (7) can be further simplified as

$$
\begin{aligned}
P_{23} & =e^{2} \Gamma_{1}^{2} \Gamma_{2} \Gamma_{3} \Delta^{2} \int \frac{d E}{2 \pi} \frac{f_{-}\left(1-f_{+}\right)}{\Delta^{2}-E^{2}} \times\left|G_{11}^{r} G_{22}^{a}-G_{12}^{r} G_{12}^{a}\right|^{2} \\
& =\frac{4 e^{2} \Gamma_{2} \Gamma_{3}}{\left(\Gamma_{2}+\Gamma_{3}\right)^{2}} \int \frac{d E}{2 \pi} f_{-}\left(1-f_{+}\right) T_{A}(E)\left[1-T_{A}(E)\right], \quad(10)
\end{aligned}
$$

where $T_{A}(E)=\Gamma_{1}^{2} G_{12}^{r} G_{12}^{a}$ is the Andreev-reflection coefficient and $f_{ \pm}(E)=f(E \pm e V)$. Just as we expected, Eq. (10) is a positive quantity. To get more physical insight, we will assume that $e V$ are small enough and we will keep only the first order in $V$ in Eq. (10). We have

$$
\begin{aligned}
P_{23}= & \frac{\Gamma_{1}^{2} \Gamma_{2} \Gamma_{3} e^{3} V}{\pi\left[\epsilon_{0}^{2}+\frac{\Gamma_{1}^{2}}{4}+\frac{\left(\Gamma_{2}+\Gamma_{3}\right)^{2}}{4}\right]^{4}} \\
& \times\left\{\epsilon_{0}^{4}+\frac{\epsilon_{0}^{2}\left[\Gamma_{1}^{2}+\left(\Gamma_{2}+\Gamma_{3}\right)^{2}\right]}{2}+\frac{\left[\Gamma_{1}^{2}-\left(\Gamma_{2}+\Gamma_{3}\right)^{2}\right]^{2}}{16}\right\} .
\end{aligned}
$$

For $e V>\Delta$, we have to calculate $P_{23}$ numerically, which is presented in the next section.

\section{RESULTS AND DISCUSSION}

We first use Eq. (10) to calculate the cross correlation at finite voltage while keeping $e V<\Delta$. In the following, we 

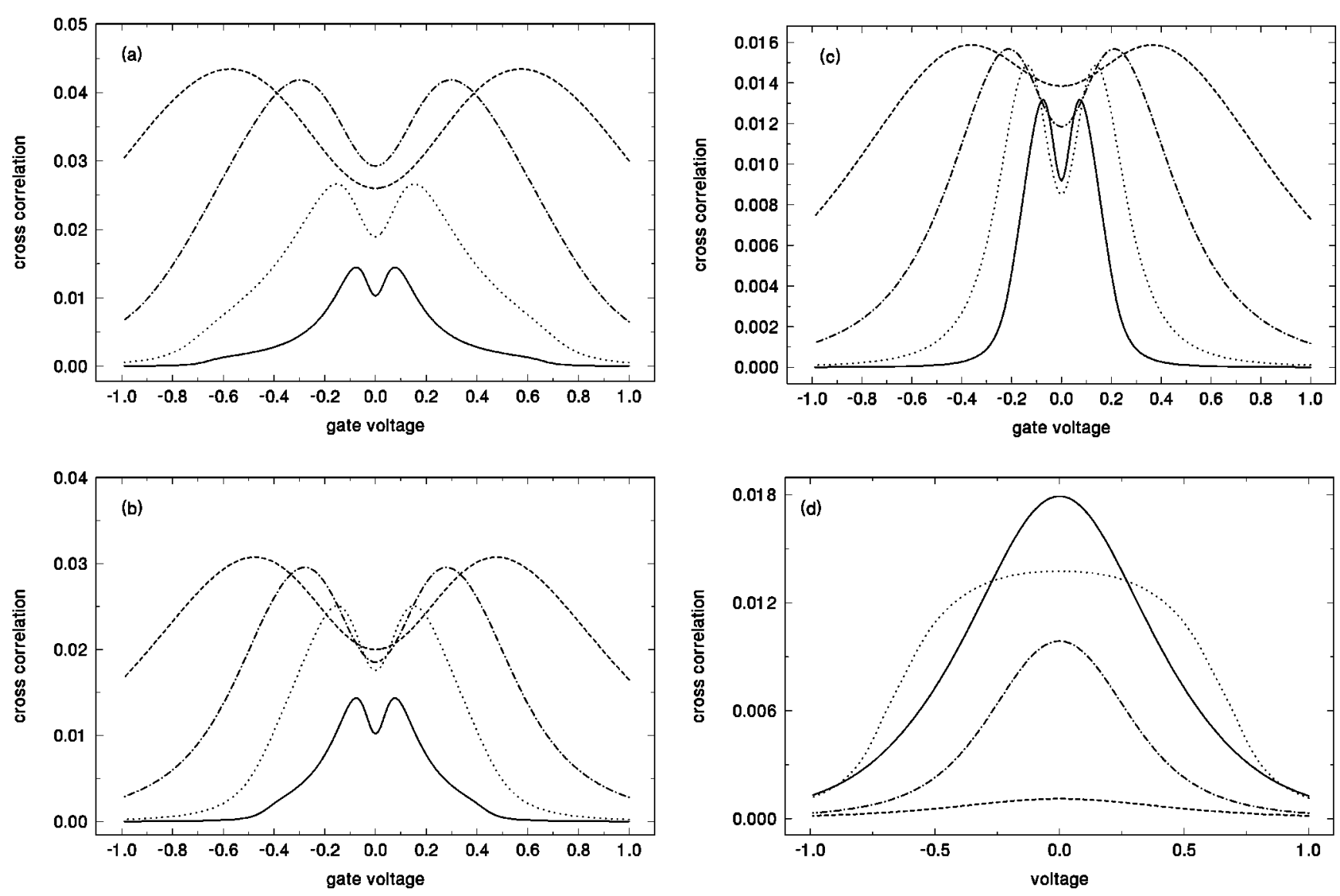

FIG. 1. The cross correlation $P_{23}$ versus gate voltage for external bias and different coupling parameters. (a) $e V=0.6$. Solid line: $\Gamma$ $=\Gamma_{1}=\Gamma_{2}=0.1$, dotted line: $\Gamma=0.2$, dot-dashed line: $\Gamma=0.4$, and dashed line: $\Gamma=0.8$. (b) $e V=0.4$. Other symbols are the same as (a). (c) $e V=0.2$. Other symbols are the same as (a). (d) Solid line: $\Gamma_{1}=0.8, \Gamma_{2}=0.1$, and $e V=0.6$. Dotted line: $\Gamma_{1}=0.1, \Gamma_{2}=0.8$, and $e V$ $=0.6$. Dot-dashed line: $\Gamma_{1}=0.8, \Gamma_{2}=0.1$, and $e V=0.2$. Dashed line: $\Gamma_{1}=0.1, \Gamma_{2}=0.8$, and $e V=0.2$.

will use $\Delta$ as the unit of energy and study the symmetric case where $\Gamma_{2}=\Gamma_{3}$. In Fig. 1 we show the cross correlation versus the gate voltage at fixed external bias $e V=0.6,0.4$, and 0.2 . Four different sets of coupling constants $\Gamma$ are chosen: (i) $\Gamma=\Gamma_{1}=\Gamma_{2}=0.8,0.4,0.2$, and 0.1 . For $\Gamma=0.8$, it represents the strong coupling between leads and the quantum dot. $^{17}$ For $e V=0.6$ [Fig. 1(a)], the cross correlation (dashed line) displays two broad peaks located symmetrically at $e v_{g}= \pm 0.6$. (ii). For the weak-coupling case $\Gamma=0.1$, the cross correlation (solid line) has two sharp peaks close to $v_{g}=0.08$ and decays quickly away from it. (iii) $\Gamma=0.2$ and 0.4 (dotted line and dot-dashed line) represent the intermediate-coupling regime for the external bias $e V=0.6$. We see that the position of the peaks shift towards the origin as one decreases $\Gamma$. The general feature of the double-peak structure can be understood as follows. We notice that two terms $F_{1}=\int d E T_{A}(E)$ and $F_{2}=\int d E T_{A}^{2}(E)$ in Eq. (10) tend to cancel each other. Note that here $F_{1}$ is proportional to the current. The shape of $F_{1}$ and $F_{2}$ are dominated by the line shape of $T_{A}$ since $T_{A}^{2}$ decays much faster than $T_{A}$. As the result of different linewidths for $F_{1}$ and $F_{2}$, we thus have the double-peak structure shown in Fig. 1(a). This is illustrated in Fig. 2 for $\Gamma=0.1$. In the weak-coupling regime, the integrals $F_{1}$ and $F_{2}$ give comparable contributions with a single peak at $v_{g}=0$. However, the integral $F_{2}$ decreases faster than that of $F_{1}$ as $v_{g}$ is increased, resulting a double-peak structure for $F_{1}-F_{2}$ (see Fig. 2). Since the linewidth of cross correlation is determined by $T_{A}$ which in turn is determined by $\Gamma$, it is thus understandable that the larger the $\Gamma$, the wider the double-peak structure. Now we gradually decrease the external bias to $e V=0.4$. We see from Fig. 1(b)

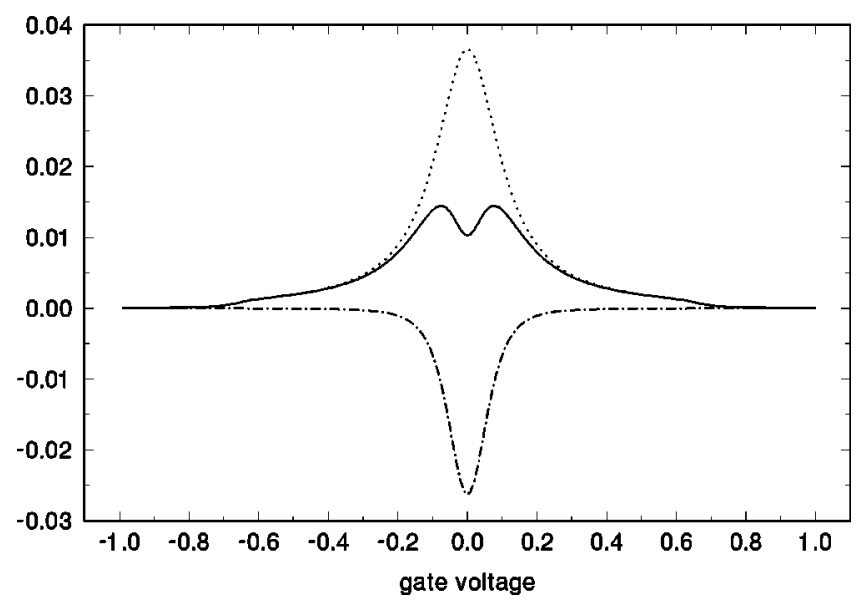

FIG. 2. The contribution of $F_{1}$ (dotted line) and $F_{2}$ (dot-dashed line) to the cross correlation $P_{23}$ (solid line) versus gate voltage at $e V=0.6$. Here $\Gamma_{1}=\Gamma_{2}=0.1$. 
that for the strong-coupling case $\Gamma=0.8$ and 0.6 , the cross correlations in the strong-coupling case still show a doublepeak structure but with smaller amplitude. We also find that the peak positions are shifted towards the origin. For the weak-coupling case $\Gamma=0.2$ and 0.1 , however, both the height and position of the peaks are roughly unchanged. As we decrease $e V$ further to 0.2 [see Fig. 1(c)], the general behavior of the cross correlation is similar to that of Fig. 1(b) except that for $\Gamma=0.2$ the peak height decreases. These behaviors can again be understood from the domination of the linewidth of $T_{A}$ in the cross correlation. For $\Gamma=0.1$, the linewidth of $T_{A}$ is about 0.16 . This means that only electrons with energy below 0.2 contribute to the cross correlation. Hence the cross correlation versus gate voltage for $\Gamma=0.1$ is the same for $e V=0.2,0.4$, and 0.6 . For $\Gamma=0.2$, the linewidth of $T_{A}$ is about 0.32 . As a result, the cross correlation remains unchanged for $e V=0.4$ and 0.6. Obviously, if we examine the cross correlation for $\Gamma=0.1$ at an even smaller external bias, e.g., $e V=0.1$, it will be different from that of $e V=0.2$. Of course, in this case, $\Gamma=0.1$ cannot be defined as the weak-coupling limit. From the above analysis, we conclude that the position of the peaks is influenced by the interplay between the external bias and the contact strength $\Gamma$. In the strong-coupling case, the position of the peak is controlled by the external bias. In the weak-coupling case, it is controlled by coupling strength $\Gamma$. Next we consider the asymmetric case when $\Gamma_{1}$ is not equal to $\Gamma_{2}$. Two sets of coupling constants are chosen. (i) $\Gamma_{1}=0.8$ and $\Gamma_{2}=0.1$. In this case, the normal lead couples strongly with the quantum dot while the superconducting leads couple weakly. Figure 1(d) shows the cross correlation (solid line for $e V=0.6$ and dot-dashed line for $e V=0.2$ ) which exhibits a single peak at $v_{g}=0$. (ii) $\Gamma_{1}=0.1$ and $\Gamma_{2}=0.8$. This is the reverse of case (i) and we see that for $e V=0.6$ (dotted line) it shows a flat region near $v_{g}=0$. We notice that the cross correlation decreases as we decrease the external bias. For the asymmetric case, the transmission coefficient $T_{A}$ is much smaller than 1 even at resonance. Therefore the contribution from $F_{2}$ is much smaller than that of $F_{1}$ resulting with just one peak for $P_{23}$ in contrast to the symmetric case. Now we examine the cross correlation versus external bias at fixed energy levels and concentrate on the following four sets of coupling constants $\Gamma_{1}=\Gamma_{2}=0.8$ and $0.1 ; \Gamma_{1}=0.8$ and $\Gamma_{2}=0.1 ; \Gamma_{1}=0.1$ and $\Gamma_{2}=0.8$. Figure 3(a) displays the cross correlation $P_{23}$ versus external voltage when $\epsilon_{0}=0$. We see that, except for $\Gamma_{1}=0.8$ and $\Gamma_{2}=0.1, P_{23}$ increases monotonically, and $P_{23}$ develops a plateau region for the other three sets of coupling parameters. These plateau regions are due to the resonant tunneling which can be seen from Fig. 3(b) where the differential cross correlation $d P_{23} / d V$ versus external voltage is depicted. Here we see typical behavior of the shot noise: ${ }^{18}$ a minimum separated by two peaks. The minimum is due to the resonant Andreev reflection since $d P_{23} / d V \sim T_{A}(1$ $\left.-T_{A}\right)$. As one increases the energy level $\left(\epsilon_{0}=0.3\right)$, the dip between two peaks can no longer reach zero, indicating that the maximum Andreev-reflection coefficient $T_{A}$ is much less than 1 . We also note that for $\Gamma_{1}=0.1$ and $\Gamma_{2}=0.8$, only one peak is left and the resonant feature has disappeared.
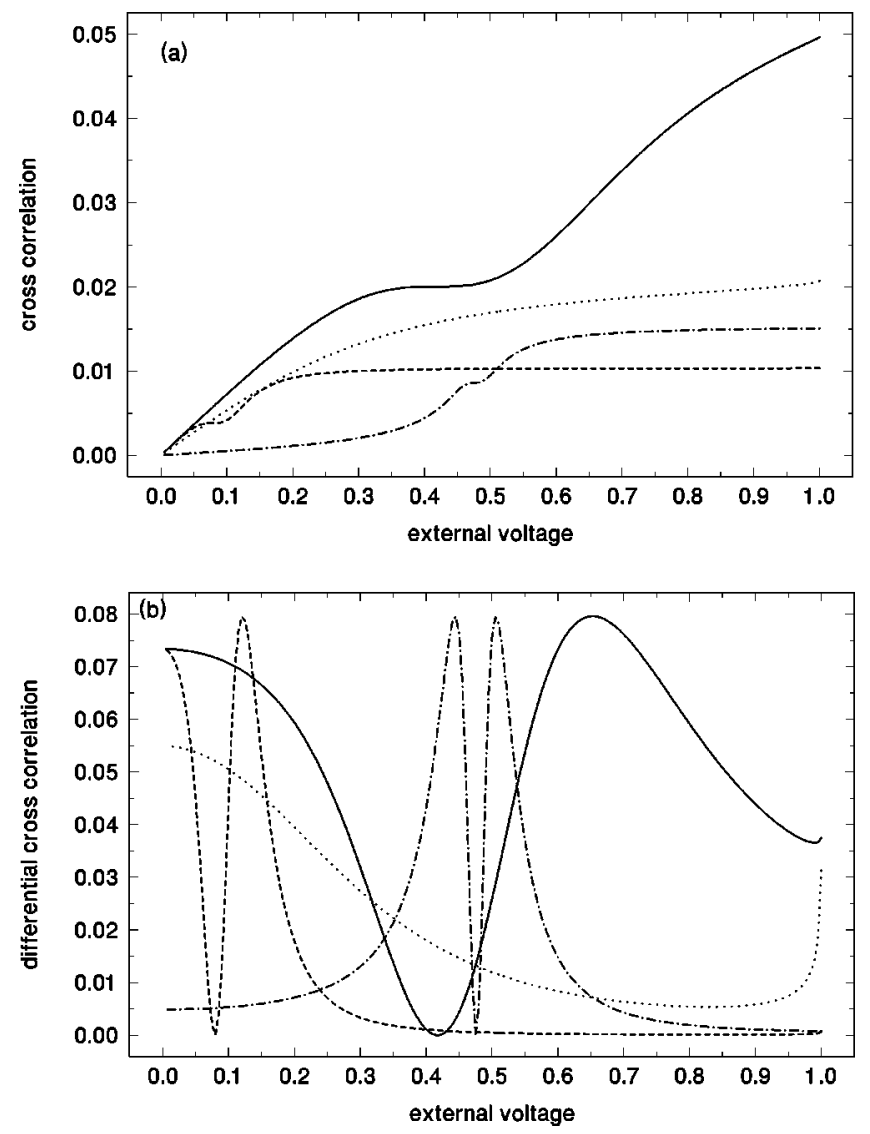

FIG. 3. (a) The cross correlation versus external voltage at $\epsilon_{0}$ $=0.0$. (b) The differential cross correlation versus external voltage at $\epsilon_{0}=0.0$. The symbols are solid line: $\Gamma_{1}=\Gamma_{2}=0.8$; dotted line: $\Gamma_{1}=0.8, \Gamma_{2}=0.1$; dot-dashed line: $\Gamma_{1}=0.1, \Gamma_{2}=0.8$; and dashed line: $\Gamma_{1}=0.1, \Gamma_{2}=0.1$.

To study the effect of quasiparticles when $e V>\Delta$, we calculate the cross correlation using Eq. (7). Figure 4 shows the cross correlation versus external voltage at $\epsilon_{0}=0.0$. We see that once the voltage is larger than the gap energy $\Delta$, the cross correlation decreases quickly, indicating fermionic contributions. For the strong coupling case $\Gamma_{1}=\Gamma_{2}=0.8, P_{23}$ becomes negative in the large $V$ limit while it remains positive for the other coupling strength. This can be understood as follows. When $e V>\Delta$, electrons with energy less than $e V$ will all participate in transport. In particular, for incoming electrons with energy inside the superconducting gap, only the two-electron current is allowed and hence the contribution to the cross correlation should be positive as we just discussed above. However, when the energy of incoming electrons is outside of the gap the current comes from of four processes: $^{10,19}$ (i) Andreev reflection, (ii) the conventional electron tunneling through the system, (iii) a "branch crossing" process ${ }^{19}$ in which an electron incident from the normal lead converts into a hole such as in the superconducting leads, and (iv) an electron (or a hole) incident from the normal lead tunnels into the superconducting lead, picks up a quasiparticle (or a quasihole) in the superconducting lead, and creates (or annihilates) a Cooper pair. In these processes, the latter three give negative contributions to the cross cor- 


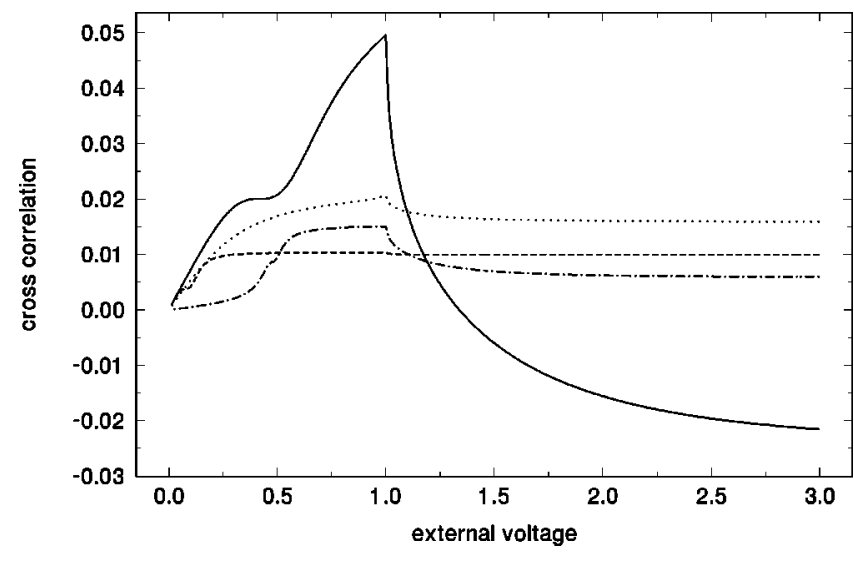

FIG. 4. The cross correlation versus external voltage at $\epsilon_{0}$ $=0.0$. The coupling parameters and corresponding symbols are the same as in Fig. 3.

relation. Competition between the Andreev-reflection process and the rest of three processes gives rise either to positive or negative cross correlation depending on which process dominates (see Fig. 4). Typically, near the resonance the Breit-Wigner form for the Andreev-reflection coefficient reads $^{20-22}$

$$
T_{A}=\frac{\Gamma_{1}^{2} \Gamma_{2}^{2}}{4\left(E^{2}-\epsilon_{0}^{2}+\Gamma \delta \Gamma / 4\right)^{2}+\Gamma_{1}^{2} \Gamma_{2}^{2}+\epsilon_{0}^{2}(\Gamma+\delta \Gamma)^{2}}
$$

and the transmission coefficient for the normal tunneling process is

$$
T=\frac{\Gamma_{1} \Gamma_{2}}{\left(E-\epsilon_{0}\right)^{2}+\Gamma^{2} / 4},
$$

where $\Gamma=\Gamma_{1}+\Gamma_{2}$ and $\delta \Gamma=\Gamma_{1}-\Gamma_{2}$. We see that the Andreev reflection is suppressed when off resonance. Furthermore, at large external bias if the resonant energy is outside the gap, the Andreev reflection is drastically suppressed and normal tunneling is allowed at a certain energy. Therefore, we expect negative cross correlation in this case. In Fig. 5, we depict $P_{23}$ versus $V$ at $\epsilon_{0}=2.0$. Since the resonant level is outside the gap, the plateau region for $P_{23}$ when $e V$ is inside the gap disappears. We see that, except for the case of $\Gamma_{1}$ $=0.8$ and $\Gamma_{2}=0.1, P_{23}$ becomes negative at large voltages. Our numerical result shows that at even larger $\epsilon_{0}$, the transport of quasiparticles dominates and all $P_{23}$ are negative at large external voltage. Note that for Fig. 4, the resonant energy of the dot is chosen at the superconducting condensate $\mu_{s}=0$ whereas in Fig. 5 the resonant energy is chosen such that it is above the superconducting gap energy. Hence, if we are in the resonant tunneling regime (weak-coupling case) then the quasiparticle current will be very small (far off the resonance) for Fig. 4 and much larger (on resonance) in Fig. 5 since quasiparticle current can exist only for $E>\Delta$. Looking at Fig. 4, only large $\Gamma=0.8$ (solid line) corresponds to the nonresonant tunneling case, hence has a larger quasiparticle current, and dominates at large voltage resulting in a negative cross correlation. Finally, we plot in Fig. 6 the $P_{23}$

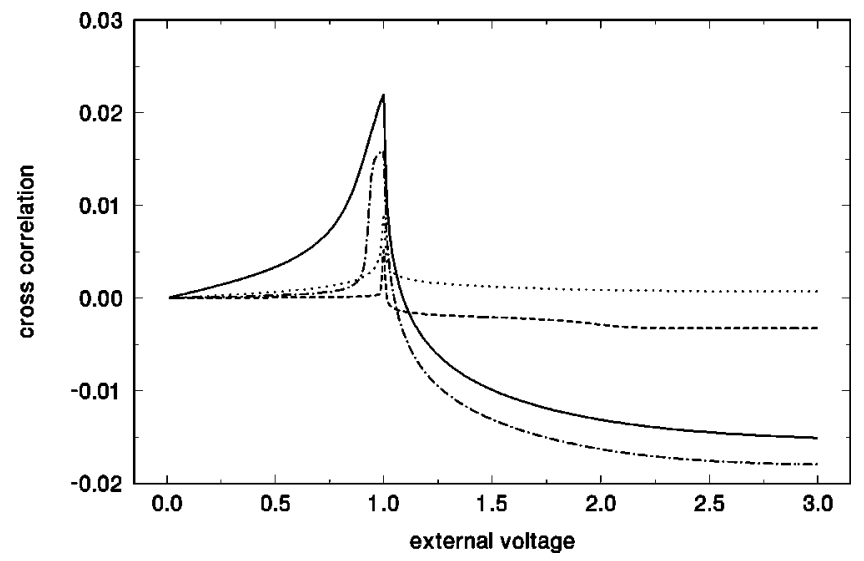

FIG. 5. The cross correlation versus external voltage at $\epsilon_{0}$ $=2.0$. The coupling parameters and corresponding symbols are the same as in Fig. 3. For illustration purposes, we have multiplied the cross correlation by a factor of 10 for the dotted line, 5 for the dot-dashed line, and 50 for the dashed line.

versus $v_{g}$ at $e V=4$. We see that at large voltages, all the cross correlation functions become negative. For the strongcoupling case, we observe oscillations of $P_{23}$ between bosonic and fermionic behaviors due to the competition between the current due to Andreev reflection and that of the quasiparticles. This can be easily checked experimentally by changing the gate voltage.

In summary, we have proposed a HBT-type experiment by using the three-terminal N-S-S hybrid mesoscopic system. When the external voltage is less than the gap energy, only a two-electron current is present. The cross correlation is found to be positive, which demonstrates bosonic behavior. However, when the external voltage is larger than the gap energy the quasiparticle will participate in the transport which gives the fermionic contribution to the cross correlation. As the result of competition between the Andreevreflection process and the other tunneling process involving quasiparticles, the cross correlation can be either positive or negative depending on which one dominates. For the strongcoupling case and at large external voltage, the cross corre-

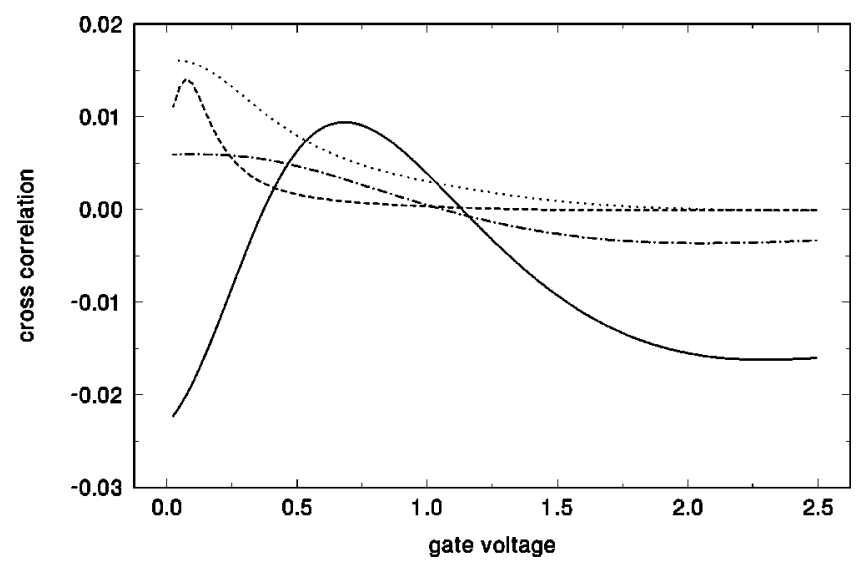

FIG. 6. The cross correlation versus gate voltage at $e V=4.0$. The coupling parameters and corresponding symbols are the same as in Fig. 3. 
lation function changes sign as one varies the gate voltage which controls the position of the resonant level. Finally it would be nice to consider the effect of the phase difference between the two superconducting leads which would enable one to predict what happens when the proximity effect is "turned off."

*Email address: jianwang@hkusub.hku.hk

${ }^{1}$ L. D. Landau and E. M. Lifshitz, Statistical Physics (Pergamon, Oxford, 1980).

${ }^{2}$ Ya. M. Blanter and M. Buttiker, Phys. Rep. 336, 2 (2000).

${ }^{3}$ R. Hanbury Brown and R. Q. Twiss, Nature (London) 177, 27 (1956).

${ }^{4}$ M. Henny et al., Science 284, 296 (1999).

${ }^{5}$ W. D. Oliver et al., Science 284, 299 (1999).

${ }^{6}$ J. Torriès and Th. Martin, Eur. Phys. J. B 12, 319 (1999).

${ }^{7}$ P. Samuelsson and M. Büttiker, Phys. Rev. B 89, 046601 (2002).

${ }^{8}$ J. Borlin, W. Belzig, and C. Bruder, Phys. Rev. Lett. 88, 197001 (2002).

${ }^{9}$ K. E. Nagaev, Phys. Rev. B 64, 081304 (2001).

${ }^{10}$ J. C. Cuevas, A. Martin-Rodero, and A. Levy Yeyati, Phys. Rev. B 54, 7366 (1996).

${ }^{11}$ Q. F. Sun, J. Wang, and T. H. Lin, Phys. Rev. B 59, 3831 (1999).

${ }^{12}$ Q. F. Sun, B. G. Wang, J. Wang, and T. H. Lin, Phys. Rev. B 61, 4754 (2000).

\section{ACKNOWLEDGMENTS}

We gratefully acknowledge support by a RGC grant from the SAR Government of Hong Kong under Grant No. HKU 7091/01P and a CRCG grant from the University of Hong Kong.

${ }^{13}$ J. Rammer and H. Smith, Rev. Mod. Phys. 58, 323 (1986).

${ }^{14}$ A. P. Jauho et al., Phys. Rev. B 50, 5528 (1994).

${ }^{15}$ J. Wang, Y. D. Wei, H. Guo, Q. F. Sun, and T. H. Lin, Phys. Rev. B 64, 104508 (2001).

${ }^{16}$ Here the density of states in the Green's function has been absorbed in the linewidth function $\Gamma$.

${ }^{17}$ Here the strong-coupling case is defined when $\Gamma>e V$ and $\Gamma$ $<e V$ corresponds to the weak-coupling case.

${ }^{18}$ Y. D. Wei, B. G. Wang, J. Wang, and H. Guo, Phys. Rev. B 60, 16900 (1999).

${ }^{19}$ G. E. Blonder, M. Tinkham, and T. M. Klapwijk, Phys. Rev. B 25, 4515 (1982).

${ }^{20}$ C. W. J. Beenakker, Rev. Mod. Phys. 69, 731 (1997).

${ }^{21}$ N. R. Claughton, M. Leadheater, and C. J. Lambert, J. Phys.: Condens. Matter 7, 8757 (1995).

${ }^{22}$ Y. D. Wei, J. Wang, H. Guo, H. Mehrez, and C. Roland, Phys. Rev. B 63, 195412 (2001). 